\title{
Review of: "Detecting Phytophthora cinnamomi associated with dieback disease on Carya cathayensis using loop-mediated isothermal amplification"
}

\author{
Bing Song Zheng
}

Potential competing interests: The author(s) declared that no potential competing interests exist.

Chinese hickory (Carya cathayensis Sarg.) is an economically and ecologically important nut plant in China. Dieback and basal stem necrosis have significantly affected plant growth and nut production. The author conducted a survey to evaluate the disease incidence at five sites in Linan County, China. The results showed that the highest incidence was recorded at the Tuankou site at up to $11.39 \%$ in 2019 . The oomycete, Phytophthora cinnamomi, was isolated from symptomatic plant tissue and plantation soil using baiting and selective media-based detection methods and identified. Artificial infection with the representative $P$. cinnamomi ST402 isolate produced vertically elongated discolorations in the outer xylem and necrotic symptoms in C. cathayensis seedlings in a greenhouse trial. LAMP detection showed a high coherence level with the baiting assays for P. cinnamomi detection in the field. This study provides the evidence of existence of high-pathogenic P. cinnamomi in the C. cathayensis plantation soil in China and the insights into a convenient tool developed for conducting field monitoring of this aggressive pathogen. The research was innovative and original. I think this paper will make a nice contribution to the journal. 